\title{
High plasma exposure to pemetrexed leads to severe hyponatremia in patients with advanced non small cell lung cancer receiving pemetrexed-platinum doublet chemotherapy
}

\author{
This article was published in the following Dove Press journal: \\ Cancer Management and Research \\ 4 June 2014 \\ Number of times this article has been viewed
}

\author{
Vikram Gota' \\ Krunal Kavathiya' \\ Kartik Doshi' \\ Murari Gurjar' \\ Solai E Damodaran' \\ Vanita Noronha ${ }^{2}$ \\ Amit Joshi \\ Kumar Prabhash ${ }^{2}$ \\ 'Department of Clinical \\ Pharmacology, Advanced Centre \\ for Treatment Research and \\ Education in Cancer, ${ }^{2}$ Department \\ of Medical Oncology, Tata Memorial \\ Hospital, Mumbai, India
}

\begin{abstract}
Background: Pemetrexed-platinum doublet therapy is a standard treatment for stage IIIb/IV nonsquamous non small cell lung cancer (NSCLC). While the regimen is associated with several grade $\geq 3$ toxicities, hyponatremia is not a commonly reported adverse effect. Here we report an unusually high incidence of grade $\geq 3$ hyponatremia in Indian patients receiving pemetrexed-platinum doublet, and the pharmacological basis for this phenomenon.

Methods: Forty-six patients with advanced NSCLC were enrolled for a bioequivalence study of two pemetrexed formulations. All patients received the pemetrexed-platinum doublet for six cycles followed by single-agent pemetrexed maintenance until progression. Pharmacokinetic blood samples were collected at predefined time points during the first cycle and the concentration-time profile of pemetrexed was investigated by noncompartmental analysis. Hyponatremic episodes were investigated with serum electrolytes, serum osmolality, urinary sodium, and urine osmolality.
\end{abstract}

Results: Sixteen of 46 patients ( $35 \%$ ) had at least one episode of grade $\geq 3$ hyponatremia. Twentyfour episodes of grade $\geq 3$ hyponatremia were observed in 200 cycles of doublet chemotherapy. Plasma exposure to pemetrexed was significantly higher in patients with high-grade hyponatremia than in those with low-grade or no hyponatremia ( $P=0.063$ and $P=0.001$, respectively). Pemetrexed clearance in high-grade hyponatremia was quite low compared with normal and low-grade hyponatremia ( $P=0.001$ and $P=0.055$, respectively). Median pemetrexed exposure in this cohort was much higher than that reported in the literature from Western studies.

Conclusion: Higher exposure to pemetrexed is associated with grade $\geq 3$ hyponatremia. The pharmacogenetic basis for higher exposure to pemetrexed in Indian patients needs further investigation.

Keywords: non small cell lung carcinoma, platinum compounds, pharmacokinetics, pharmacogenetics

\section{Introduction}

The platinum-based doublet is the chemotherapy of choice in patients with stage IIIb/IV non small cell lung carcinoma (NSCLC). ${ }^{1}$ Pemetrexed is given with platinum compounds in patients with the nonsquamous type of NSCLC. ${ }^{2}$ The pemetrexed-platinum combination has an overall survival advantage in patients with NSCLC. ${ }^{3}$ However, this combination is known to cause grade $3 / 4$ adverse drug reactions, including neutropenia, thrombocytopenia, anemia, nausea, vomiting, rash, and diarrhea. ${ }^{4}$

Hyponatremia is not a common adverse drug reaction associated with the pemetrexed-platinum combination. Cisplatin is associated with grade $3 / 4$ hyponatremia
Correspondence: Kumar Prabhash Department of Medical Oncology, Tata Memorial Hospital, Dr. E Borges Road, Parel, Mumbai 4000I2, India

Tel +9l 2227405130

Fax +9| 222740506 I

Email kumarprabhashtmh@gmail.com 
in $4 \%-10 \%$ of patients due to renal salt wasting. ${ }^{5}$ However, the incidence of severe hyponatremia with carboplatin is much lower, and mostly due to the syndrome of inappropriate antidiuretic hormone secretion (SIADH). There are very few reports of pemetrexed-induced hyponatremia. According to one report, the incidence of hyponatremia was around $2.12 \%$ in pemetrexed-treated patients. ${ }^{6}$ However, for reasons that are not well understood, a high incidence of hyponatremia has been observed in our patients receiving a pemetrexedplatinum combination for NSCLC. This trend was further confirmed during a bioequivalence study of two pemetrexed formulations conducted in our center. Here we report the findings of our exploratory study to understand the reasons for the high incidence of hyponatremia observed in our patients, particularly its correlation with pemetrexed exposure.

\section{Materials and methods}

Forty-six patients with NSCLC were enrolled for the bioequivalence study (Clinical Trials Registry of India, CTRI/2012/09/002972) of pemetrexed (Pemgem ${ }^{\mathrm{TM}}$, Dr Reddy's Laboratories Ltd, Hyderabad, India) versus Alimta ${ }^{\circledR}$ (Eli Lilly and Company, Indianapolis, IN, USA) in combination with platinum compounds. Patients received pemetrexed $500 \mathrm{mg} / \mathrm{m}^{2}$ with either cisplatin $75 \mathrm{mg} / \mathrm{m}^{2}$ or a dose of carboplatin based on a target area under the curve (AUC) of 5 . Pemetrexed was reconstituted in $100 \mathrm{~mL}$ of normal saline and administered as an intravenous infusion over 10 minutes, followed by administration of cisplatin in normal saline or carboplatin in 5\% dextrose solution over 30-60 minutes. The study was approved by the institutional review board at our institution. Written informed consent was obtained from all patients prior to enrolling them in the bioequivalence study. Patients received the pemetrexed formulation of their choice.

Pharmacokinetic blood samples were collected in the first cycle during and at various time points after the 10-minute pemetrexed infusion. The safety analysis comprised blood counts, serum electrolytes, and liver and renal function tests as per routine institutional practice. Owing to a high incidence of hyponatremia in the first few patients, it was decided to do a complete work-up comprising serum osmolality, a urine sodium spot test, and urine osmolality in subsequent patients whenever a hyponatremia episode was observed. Hyponatremia was managed as per standard institutional practice. The number of episodes of grade $\geq 3$ hyponatremia over six cycles and number of patients requiring intervention in the form of salt tablets or normal saline injections were evaluated. After six cycles of the doublet regimen, patients were administered single-agent pemetrexed as maintenance treatment according to institutional practice. Hyponatremia was graded according to Common Terminology Criteria for Adverse Events, version 4.03.

\section{Pharmacokinetics of pemetrexed}

Plasma pemetrexed levels were measured using a validated high-performance liquid chromatography assay. The AUC of pemetrexed and its clearance was estimated by noncompartmental analysis using WinNonlin software (WinNonlin Professional, version 6.3, Certara USA, Inc., St. Louis, MO, USA).

\section{Statistical analysis}

Serum sodium levels were further classified as normal (131-145 mEq/L), low-grade hyponatremia (125-130 mEq/L), or high-grade hyponatremia ( $\leq 124 \mathrm{mEq} / \mathrm{L})$. Mean values for AUC and clearance was compared between the groups using the nonparametric Mann-Whitney $U$ test. The tendency for hyponatremia with respect to pemetrexed exposure (AUC) in the three groups was analyzed using the Kruskal-Wallis test. The effect of covariates on hyponatremia was analyzed using logistic regression with backward elimination. A $P$-value less than 0.05 was considered to be statistically significant.

\section{Results}

We prospectively evaluated 46 patients with NSCLC who received the pemetrexed-platinum doublet regimen. Sixteen of 46 patients developed grade $\geq 3$ hyponatremia at least once during the doublet regimen. The baseline characteristics of patients having hyponatremia and those without are shown in Table 1. Logistic regression analysis showed that baseline variables including age, sex, stage of disease, performance status, formulation (Pemgem or Alimta), or regimen (cisplatin or carboplatin) did not have any effect on hyponatremia.

Most of the hyponatremia cases were reported during first cycle of chemotherapy. There was a total of 26 episodes of grade $\geq 3$ hyponatremia over 200 chemotherapy cycles of the doublet regimen. The median number of cycles of chemotherapy administered was six (range 1-6). Four patients were treated with saline injection for hyponatremia. Median chloride levels were 91 (84-94) mEq/L, while potassium was 4.5 (3.58-5.4) mEq/L. Almost all patients had borderline low chloride $(n=14)$ and normal potassium $(n=16)$ levels. Median serum osmolality was 273 (256-286) $\mathrm{mOsmol} / \mathrm{kg}$ while urine osmolality was 401 (219-635) $\mathrm{mOsmol} / \mathrm{kg}$ and fractional excretion of sodium in urine was $31(9-93) \mathrm{mEq} / \mathrm{L}$ (Table 2). Serum creatinine did not change significantly from 
Table I Baseline characteristics

\begin{tabular}{|c|c|c|}
\hline Characteristics & $\begin{array}{l}\text { Hyponatremic } \\
\text { patients } \\
(\mathrm{n}=16)\end{array}$ & $\begin{array}{l}\text { Patients not having } \\
\text { hyponatremia } \\
(\mathrm{n}=30)\end{array}$ \\
\hline \multicolumn{3}{|l|}{ Sex } \\
\hline Male & 12 (75\%) & $19(63 \%)$ \\
\hline Female & $4(25 \%)$ & II (37\%) \\
\hline \multicolumn{3}{|l|}{ Age } \\
\hline$<60$ years & $9(56 \%)$ & $21(70 \%)$ \\
\hline$>60$ years & $7(44 \%)$ & $9(30 \%)$ \\
\hline \multicolumn{3}{|l|}{ Stage } \\
\hline Grade 3 & I (6\%) & $2(7 \%)$ \\
\hline Grade 4 & 15 (94\%) & $28(93 \%)$ \\
\hline \multicolumn{3}{|l|}{ ECOG PS score } \\
\hline 1 & $13(81 \%)$ & 27 (90\%) \\
\hline 2 & $3(19 \%)$ & $3(10 \%)$ \\
\hline \multicolumn{3}{|c|}{ Pemetrexed formulation } \\
\hline Pemgem $^{\mathrm{TM}}$ & $13(8 \mid \%)$ & $21(70 \%)$ \\
\hline Alimta $^{\circledR}$ & $3(19 \%)$ & $9(30 \%)$ \\
\hline \multicolumn{3}{|l|}{ Platinum regimen } \\
\hline Cisplatin & $2(12.5 \%)$ & $5(17 \%)$ \\
\hline Carboplatin & 14 (87.5\%) & $25(83 \%)$ \\
\hline
\end{tabular}

Notes: Pemgem ${ }^{\text {TM }}$ (Dr Reddy's Laboratories Ltd, Hyderabad, India); Alimta ${ }^{\circledast}$ (Eli Lilly and Company, Indianapolis, IN, USA).

Abbreviations: ECOG, Eastern Cooperative Oncology Group; PS, performance status.

baseline during the course of treatment, even in those patients who developed hyponatremia.

\section{Correlation between pemetrexed AUC, clearance, and hyponatremia}

Patients with high-grade hyponatremia had a higher AUC compared with patients having normal or low-grade hyponatremia ( $P=0.001$ and $P=0.063$, respectively). There was a trend towards progressively more severe hyponatremia with higher exposure to pemetrexed (Kruskal-Wallis, $P=0.006$, Figure 1).

Consequently, the clearance of pemetrexed was less in patients with high-grade hyponatremia compared with those with normal/low grade hyponatremia $(P=0.001$ and $P=0.055$, respectively). Clearance of pemetrexed (median and range) in the normal, low-grade, and high-grade groups was 4,021 $(1,462-11,698) \mathrm{mL}$ per hour, 3,096 $(2,022-7,336) \mathrm{mL}$ per hour, and 1,569 $(638-2,503) \mathrm{mL}$ per hour, respectively (Kruskal-Wallis, $P=0.007$ ).

\section{Maintenance pemetrexed therapy}

Eighteen patients received maintenance pemetrexed therapy. Three of 18 patients on maintenance pemetrexed developed grade $\geq 3$ hyponatremia, one of whom was a 25 -year-old woman diagnosed with metastatic papillary adenocarcinoma who developed grade 4 hyponatremia (sodium $103 \mathrm{mg} / \mathrm{dL}$ ) on day 4 of the fifth maintenance cycle. Other significant biochemical findings included low levels of serum chloride ( $88 \mathrm{mEq} / \mathrm{L})$, low serum osmolality (263 mOsmol/ $/ \mathrm{kg})$, high urine osmolality ( $889 \mathrm{mOsmol} / \mathrm{kg}$ ), and high urine random sodium $(170 \mathrm{mEq} / \mathrm{L})$. Serum potassium, magnesium, and calcium were within normal limits, and the patient was asymptomatic. She was admitted to the intensive care unit and treated with intravenous hypertonic saline. Sodium and other parameters returned to baseline by day 7 and the patient was discharged.

Table 2 Serum and urine electrolytes and osmolality profile in patients suffering from hyponatremia $(n=16)$

\begin{tabular}{|c|c|c|c|c|c|}
\hline \multicolumn{3}{|c|}{ Serum electrolytes } & \multirow{2}{*}{$\begin{array}{l}\text { Serum } \\
\text { osmolality }\end{array}$} & \multirow{2}{*}{$\begin{array}{l}\text { Urine } \\
\text { osmolality }\end{array}$} & \multirow{2}{*}{$\begin{array}{l}\text { Urinary } \\
\text { sodium }\end{array}$} \\
\hline Sodium & Potassium & Chloride & & & \\
\hline 127 & 4.6 & 92 & NA & NA & NA \\
\hline 128 & 5.3 & 93 & NA & NA & NA \\
\hline 129 & 3.58 & 91 & NA & NA & NA \\
\hline 117 & 4.2 & 87 & NA & NA & NA \\
\hline 127 & 4.5 & 91 & NA & NA & NA \\
\hline 129 & 4.3 & 93 & 268 & 635 & NA \\
\hline 124 & 4.49 & NA & 276 & 470 & 25 \\
\hline 122 & 5.4 & 88 & 256 & 442 & 60 \\
\hline 127 & 4.5 & 90 & 258 & 401 & 54.6 \\
\hline 128 & 4.7 & 94 & 276 & 491 & 15 \\
\hline 124 & 4.5 & 90 & 272 & 312 & 39 \\
\hline 129 & 4.3 & 96 & 273 & 219 & 9 \\
\hline 122 & 3.85 & 84 & 258 & 309 & 16 \\
\hline 128 & 4.86 & 93 & 277 & 168 & 13 \\
\hline 128 & 4.05 & 93 & 286 & 258 & 37 \\
\hline 122 & 5.3 & 87 & 276 & 471 & 93 \\
\hline
\end{tabular}

Notes: Normal values are $135-145 \mathrm{mEq} / \mathrm{L}$ for sodium, $3.5-4.5 \mathrm{mEq} / \mathrm{L}$ for potassium, $98-108 \mathrm{mEg} / \mathrm{L}$ for chloride, $40-1,000 \mathrm{mOsmol} / \mathrm{L}$ for urine osmolality, and 275-285 mOsmol/L for serum osmolality.

Abbreviation: NA, not available. 


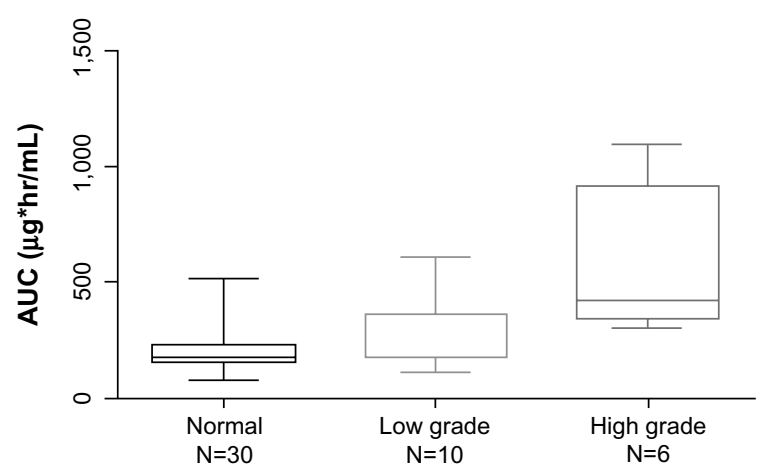

Figure I Box-and-whisker plot showing higher AUC values in patients with highgrade hyponatremia compared with normal and low-grade hyponatremia (normal | 3 |-| 45 mEq/L; low-grade I25-130 mEq/L; high-grade < I 25 mEq/L).

Abbreviation: AUC, area under the curve.

\section{Discussion}

In the present study, an unusually high incidence (35\%) of hyponatremia was seen in NSCLC on treatment with a pemetrexed-platinum combination. Such a high incidence of severe hyponatremia has not been reported in the past. Chee et al reported a $3 \%$ incidence of grade $\geq 3$ hyponatremia due to the pemetrexed-carboplatin combination. ${ }^{7}$ Their study enrolled a similar number of patients as in our study. In another study, the incidence of hyponatremia was $0.5 \% .{ }^{8}$ The high incidence of hyponatremia in Indian patients suggests possible ethnic variations in the disposition and off-target effects of the doublet.

Although hyponatremia is not a very common side effect of carboplatin or pemetrexed, carboplatin is known to cause severe hyponatremia occasionally, mostly due to SIADH. ${ }^{9}$ Case reports have shown that carboplatin can cause SIADH by increasing the activity of vasopressin-associated peptide. Cisplatin on the other hand causes hyponatremia by causing direct damage to renal tubular cells, resulting in renal salt wasting. ${ }^{10}$ SIADH and renal salt wasting are biochemically identical conditions that can only be differentiated on clinical grounds. Euvolemia or mild hypervolemia is seen in SIADH, as opposed to hypovolemia in renal salt wasting. Differentiation of these two conditions is essential, because treatment requires fluid restriction in SIADH and fluid and electrolyte replenishment in renal salt wasting. In our study, spot urine sodium excretion was higher in patients with hyponatremia and urine osmolality was higher than the serum osmolality, suggesting SIADH as the likely mechanism of hyponatremia. Further studies that include assessment of antidiuretic hormone are required to confirm this hypothesis. The severity of hyponatremia had a direct correlation with exposure to pemetrexed. Pemetrexed exposure (AUC) in high-grade hyponatremia patients was reported to be $427(305-1,095) \mu \mathrm{g} \cdot$ hour $/ \mathrm{mL}$, which is significantly higher than the AUC of $182(99.8-302) \mu \mathrm{g} \cdot$ hour/mL reported in Western studies. ${ }^{11}$ It is therefore conceivable that higher exposure to pemetrexed is responsible for its hyponatremia effects in Indian patients.

The high incidence of hyponatremia observed during single-agent maintenance therapy lends further credence to the fact that pemetrexed by itself may have hyponatremic effects, independent of the coadministered platinum. Interestingly, only one of the three patients had hyponatremia during the earlier doublet regimen. Clearance of pemetrexed is known to decrease with prolonged treatment, ${ }^{12}$ which might have resulted in higher exposure during maintenance chemotherapy in the other two cases. ${ }^{12}$

Pemetrexed is predominantly excreted by glomerular filtration and renal secretion. It is excreted by organic anion transporter 3 (OAT3) in the proximal tubule. The frequency of the OAT3 allele in the Western population is approximately $1 \%$. In vitro assay with three variants ( $p$. Arg149Ser, p. Gln239Stop, and p. Ile260Arg) showed complete loss of function. ${ }^{13}$ However, allele frequency has not been reported in the Indian population. ${ }^{13}$ Future studies should look at OAT3 polymorphisms in Indian patients because this could explain the increased exposure to and reduced clearance of pemetrexed observed in most subjects who develop hyponatremia.

This study has an obvious limitation, ie, the correlation between pemetrexed exposure and hyponatremia was an incidental finding. Knowledge of platinum exposure in this cohort of patients would have given a better understanding of the nature of the drug-drug interaction between pemetrexed and platinum, if any. The plasma levels of pemetrexed may be suggestive but do not prove causation, since we do not know the plasma levels of the platinum compounds. This potential interaction needs to be evaluated in prospectively designed clinical trials in the future.

\section{Conclusion}

We report a high incidence of severe hyponatremia in Indian patients with NSCLC receiving the pemetrexedplatinum combination. Higher exposure to pemetrexed was found in patients with hyponatremia than in those without hyponatremia. The pharmacogenetic basis of this phenomenon in Indian patients needs to be investigated.

\section{Disclosure}

This work was partially supported by Dr Reddy's Laboratories, Hyderabad, India. The authors report no other conflicts of interest in this work. 


\section{References}

1. D'Addario G, Pintilie M, Leighl NB, Feld R, Cerny T, Shepherd FA. Platinum-based versus non-platinum-based chemotherapy in advanced non-small-cell lung cancer: a meta-analysis of the published literature. J Clin Oncol. 2005;23:2926-2936.

2. Lee HY, Ahn MJ, Park YH, et al. Adenocarcinoma has an excellent outcome with pemetrexed treatment in Korean patients: a prospective, multicenter trial. Lung Cancer. 2009;66:338-343.

3. Zukin M, Barrios CH, Pereira JR, et al. Randomized phase III trial of single-agent pemetrexed versus carboplatin and pemetrexed in patients with advanced non-small-cell lung cancer and Eastern Cooperative Oncology Group performance status of 2. J Clin Oncol. 2013;31:2849-2853.

4. Li M, Zhang Q, Fu P, et al. Pemetrexed plus platinum as the first-line treatment option for advanced non-small cell lung cancer: a meta-analysis of randomized controlled trials. PLoS One. 2012;7:17.

5. Berghmans T. Hyponatremia related to medical anticancer treatment. Support Care Cancer. 1996;4:341-350.

6. eHealthMe. Pemetrexed outcomes reported from 2555 patients. Available from: http://www.ehealthme.com/pemetrexed. Accessed January 5, 2014.

7. Chee CE, Jett JR, Bernath AM Jr, et al. Phase 2 trial of pemetrexed disodium and carboplatin in previously untreated extensive-stage small cell lung cancer, N0423. Cancer. 2010;116:2382-2389.
8. Socinski MA, Smit EF, Lorigan P, et al. Phase III study of pemetrexed plus carboplatin compared with etoposide plus carboplatin in chemotherapy-naive patients with extensive-stage small-cell lung cancer. J Clin Oncol. 2009;27:4787-4792.

9. Fujioka S, Nakamura H, Miwa K, et al. Syndrome of inappropriate secretion of antidiuretic hormone (SIADH) following carboplatinpaclitaxel administration in a patient with lung cancer. Pharmazie. 2011;66:729-730.

10. Liamis G, Milionis H, Elisaf M. A review of drug-induced hyponatremia. Am J Kidney Dis. 2008;52:144-153.

11. Clinical Pharmacology Review. Alimta ${ }^{\mathrm{TM}}$ (pemetrexed). Available from: http://www.fda.gov/downloads/Drugs/.../UCM252981.pdf. Accessed December 3, 2013.

12. van den Bogaert DP, Pouw EM, van Wijhe G, et al. Pemetrexed maintenance therapy in patients with malignant pleural mesothelioma. J Thorac Oncol. 2006;1:25-30.

13. Erdman AR, Mangravite LM, Urban TJ, et al. The human organic anion transporter 3 (OAT3; SLC22A8): genetic variation and functional genomics. Am J Physiol Renal Physiol. 2006;290:15.
Cancer Management and Research

\section{Publish your work in this journal}

Cancer Management and Research is an international, peer-reviewed open access journal focusing on cancer research and the optimal use of preventative and integrated treatment interventions to achieve improved outcomes, enhanced survival and quality of life for the cancer patient The journal welcomes original research, clinical \& epidemiological

\section{Dovepress}

studies, reviews \& evaluations, guidelines, expert opinion \& commentary, case reports \& extended reports. The manuscript management system is completely online and includes a very quick and fair peerreview system, which is all easy to use. Visit http://www.dovepress.com/ testimonials.php to read real quotes from published authors. 